\title{
CARACTERÍSTICAS DE NEONATOS COM SÍNDROME DO DESCONFORTO RESPIRATÓRIO CONSIDERANDO A VIA DE PARTO EM UMA UNIDADE DE TERAPIA INTENSIVA DA REGIÃO CENTRAL DO RS
}

\author{
CHARACTERISTICS OF NEONATES WITH RESPIRATORY \\ DISTRESS SYNDROME ADMITTED TO AN INTENSIVE CARE UNIT OF \\ THE CENTRAL REGION OF RS
}

\author{
Daniele Prestes ${ }^{1}$ \\ Vivian da Pieve Antunes ${ }^{2}$ \\ Dannuey Machado Cardoso ${ }^{3}$ \\ Alethéia Peters Bajotto ${ }^{4}$ \\ Adriane Schmidt Pasqualoto ${ }^{5}$
}

\section{RESUMO}

Objetivo: Analisar as características dos neonatos com Síndrome do Desconforto Respiratório (SDR), considerando a via de parto, admitidos em uma Unidade de Terapia Intensiva de um hospital universitário da região central do Rio Grande do Sul. Materiais e métodos: Estudo retrospectivo, realizado no período de abril a maio de 2017, através da análise de prontuários de neonatos com idade gestacional acima de 37 semanas, ambos os sexos, internados no ano de 2016 na Unidade de Terapia Intensiva Neonatal (UTIN) do Hospital Universitário de Santa Maria com CID 10 P22 - Desconforto (angústia) respiratório(a) do recém-nascido. Resultados: A amostra foi constituída por 40 registros de nascimentos ( 25 do sexo masculino), divididos em parto vaginal $(n=11)$ e cesárea $(n=29)$, representando $27,5 \%$ e $72,5 \%$, respectivamente, das internações totais. A média de idade materna foi de $27 \pm 7$ anos, com uma média de $7 \pm 5$ consultas pré-natais. Durante o período de internação $65 \%(n=26)$ dos 40 neonatos necessitaram de oxigenoterapia. Conclusão: A partir dos resultados obtidos no presente estudo, observamos que a SDR representa $11 \%$ das internações ocorridas na UTIN, no ano de 2016. A prevalência, quanto ao tipo de parto, foi de neonatos nascidos de cesariana, sem a presença do trabalho de parto e com idade gestacional de 38 semanas.

DESCRITORES: Unidades de Terapia Intensiva Neonatal. Recém-Nascido. Síndrome do Desconforto Respiratório. Cesárea. Parto Normal.

\begin{abstract}
Objective: To analyze the characteristics of newborns with Respiratory Distress Syndrome (RDS), considering the route of delivery, admitted to an Intensive Care Unit of a university hospital in the central region of Rio Grande do Sul, Brazil. Materials and methods: A retrospective study, conducted from April to May 2017, through the analysis of records of neonates with gestational age above 37 weeks, both sexes, hospitalized in 2016 at the Neonatal Intensive Care Unit (NICU) of the University Hospital of Santa Maria, with ICD 10 P22 - Respiratory distress syndrome of newborn. Results: The sample consisted of 40 birth records ( 25 males), divided into vaginal $(n=11)$ and caesarean $(n=29)$, representing $27.5 \%$ and $72.5 \%$, respectively, of total hospitalization. The mean maternal age was $27 \pm 7$ years, with a mean of $7 \pm 5$ prenatal consultations. During the hospitalization period, $65 \%(n=26)$ of the 40 neonates required oxygen therapy. Conclusion: Based on the results obtained in the present study, we observed that the RDS represents $11 \%$ of the hospitalizations that occurred in the NICU in 2016. The prevalence, in terms of route delivery, was of neonates born caesarean, without the presence of labor and gestational age of 38 weeks.
\end{abstract}

DESCRIPTORS: Neonatal Intensive Care Units. Newborn. Respiratory Distress Syndrome. Caesarean section. Normal birth.

1- Fisioterapeuta, Especialista em Reabilitação físico-motora e Mestranda em Distúrbios da Comunicação Humana, Universidade Federal de Santa Maria, Santa Maria, RS, Brasil.

2- Mestre em Distúrbios da Comunicação Humana pela Universidade Federal de Santa Maria, Fisioterapeuta da Unidade de Terapia Intensiva do Hospital Universitário de Santa Maria, RS, Brasil

3- Fisioterapeuta, Coordenador e Docente do Curso de Fisioterapia da Faculdade Dom Alberto, Santa Cruz do Sul, RS, Brasil. Doutorando do Programa de Pós-Graduação em Ciência Pneumológica, Universidade Federal do Rio Grande do Sul-UFRGS, Porto Alegre, RS, Brasil. 4- Fisioterapeuta, Docente do Curso de Fisioterapia do Centro Universitário Franciscano, Santa Maria, RS, Brasil.

5- Fisioterapeuta, Docente do Curso de Especialização em reabilitação físico-motora e do Programa de Pós-Graduação em Distúrbios da Comunicação Humana, Universidade Federal de Santa Maria, Santa Maria, RS, Brasil. 
$\mathrm{O}$ período neonatal é considerado o mais vulnerável para a sobrevivência compreendendo os primeiros 28 dias de vida ${ }^{1}$. Os distúrbios respiratórios estão entre as ocorrências mais comuns nas primeiras horas de vida extrauterina dos neonatos, decorrentes de diversas condições e geralmente associados ao acúmulo de líquido retido nos alvéolos e interstício pulmonar. Esse líquido é liberado pelas vias aéreas, canais linfáticos e capilares, por meio da estimulação adrenérgica que ocorre durante o processo normal do parto vaginal ${ }^{2}$.

Esse fluido pulmonar fetal desempenha o papel na manutenção do volume residual, de modo a formar a capacidade pulmonar. A reabsorção começa em torno de 2 a 3 dias antes do nascimento e a onda de epinefrina, durante o trabalho de parto, contribui, acelerando esse mecanismo ${ }^{3}$.

O trabalho de parto está diretamente relacionado na maturação final pulmonar, sendo responsável pela liberação de catecolaminas e corticosteroides que são essenciais nesse processo ${ }^{4} 2013$, at $37-38+6$ weeks of pregnancy. The women were divided into exposed group (Group A. Furzán ${ }^{5}$ corrobora afirmando que, durante o trabalho de parto, a transição fetal do meio líquido para o meio externo é mediada por um conjunto de substâncias adaptativas ao estresse, a saber: a ocitocina, a vasopressina, o cortisol, as catecolaminas e as beta-endorfinas. Assim, a elevação transitória desses hormônios pode ser considerada o primeiro passo nos processos de adaptação e maturação fisiológica do bebê, com efeitos benéficos sobre sua função respiratória, imunológica e comportamental, processo esse que não ocorre em cesarianas eletivas $^{5}$.

A Síndrome do desconforto respirató- rio (SDR) é uma desordem respiratória que ocorre, principalmente, pela deficiência de substâncias do sistema surfactante pulmonar e é caracterizada por insuficiência respiratória que tem seu início ao nascimento e pode progredir. Representa um dos problemas respiratórios em neonatos mais graves e frequentes no mundo, além da maioria dos casos de morbimortalidade nos primeiros dias de vida. Comumente, a incidência e a gravidade aumentam à proporção que diminui a idade gestacional ${ }^{6}$.

Suas principais manifestações clínicas são: taquipneia, dispneia, tiragem intercostal, cianose, batimento da asa do nariz. Além disto, representa a principal causa de internações nas unidades de terapia intensiva neonatal (UTIN). Sendo assim, a relação da SDR e a cesariana tem sido colocado em questão desde meados de 1940 e sua maior incidência, conforme estudos, está em neonatos do sexo masculino ${ }^{5,7}$ emergency room, delivery room, or during physical assessment in the newborn nursery. The authors have proposed a practical approach to diagnose and manage such infants with suggestions for consulting a neonatologist at a regional center. Their objective is that practicing pediatricians should be able to assess and stabilize such infants, and transfer to or consult a neonatologist, cardiologist, or pulmonologist after reading this article.","author":[[“dropping-particle":"',",family":"Pramanik","given":"Arun K.","non-dropping-particle":"',"parse-names":false,"suffix":"”\},\{“dropping-particle":"”,"family":"Rangaswamy","given":"Nandeesh","non-dropping-particle":"',",parse-names":false,"suffix":."'\},\{“dropping-particle".'”, ,'family":"Gates","given":"Thomas","non-dropping-par- 
ticle":"”,"parse-names":false,"suffix":"'\}],"container-title":"Pediatric Clinics of North America","id":"ITEM-2","issue":"2","issued":\{“date-parts":[["2015"]]\},"page":"453-469","publisher":"Elsevier Inc","title":"Neonatal Respiratory Distress: A Practical Approach to Its Diagnosis and Management","type":"article-journal","volume":"62"],"uris":[“http://www. mendeley.com/documents/?uuid=a770c02d-3c69-4e30-a992-26b2a527fd92"]\}],"mendeley": :"formattedCitation":"<sup >5,7<1 sup>","plainTextFormattedCitation":"5,7","previouslyFormattedCitation":"<sup $>5,7<$ /sup>"\},"properties":\{“notelndex":0\},"schema":"https://github.com/citation-style-language/ schema/raw/master/csl-citation.json"\}.

Considerando os aspectos sobre a SDR em neonatos, observa-se a necessidade de explorar as características desta população, considerando a via de parto, a fim de elucidar o que é considerado um dos problemas respiratórios mais graves e frequentes mundialmente. Sendo assim, o objetivo do estudo foi analisar as características dos neonatos com Síndrome do desconforto respiratório, considerando a via de parto, que foram admitidos em Unidade de Terapia Intensiva de um hospital universitário da Região Central do Rio Grande do Sul (RS).

\section{MATERIAIS E MÉTODOS}

O delineamento do estudo é uma análise retrospectiva em base de dados secundários, realizado na UTIN de um Hospital Universitário, na Região Central do RS, no período de abril a maio de 2017. Este estudo foi aprovado no Comitê de Ética e Pesquisa (CEP) da Universidade Federal de Santa Maria (UFSM) com número do parecer 2.009.633.
Foram incluídos prontuários de neonatos com idade gestacional acima de 37 semanas, de ambos os sexos, que foram admitidos na UTIN no ano de 2016, com CID (Classificação Internacional de Doenças) 10 P22 - Desconforto (angústia) respiratório(a) do recém-nascido e que foram internados imediatamente após ao nascimento ou em até 24 horas desde que não tenha recebido alta da maternidade. Excluiu-se os registros dos neonatos com alterações neurológicas congênitas, síndromes genéticas com influência sobre o tônus muscular e registros com histórico de anóxia ao nascimento.

Foram coletados dos prontuários, por um avaliador previamente treinado, as informações que compuseram as variáveis de interesse: dados maternos (idade, complicações gestacionais ou durante o parto, entre outros), dados neonatais no nascimento (sexo, peso, apgar, necessidade de suporte ventilatório, entre outros) e dados neonatais na internação (uso oxigenoterapia, medicações, entre outros).

Primeiramente, o número do Serviço de Arquivo Médico e Estatística (SAME) foi obtido por meio do Arquivo de Registros de Internações existentes na UTIN, que contêm dados como: data da internação, idade gestacional (IG) e patologia. Posteriormente, os prontuários foram solicitados no SAME, onde a coleta foi realizada. Os registros que não possuíam todos os dados necessários para saber sua elegibilidade (IG ou patologia da internação) foram solicitados ao SAME para verificação.

Os dados foram analisados no programa SPSS (versão 20,0) e, nas tabelas, foram apresentados em mediana (intervalo 
interquartil) e os dados categóricos foram apresentados em frequências e porcentagens. Para avaliar a normalidade foi utilizado o teste de Shapiro-Wilk. As variáveis numéricas foram comparadas entre os grupos pelos testes de Mann-Whitney. Já as variáveis nominais foram comparadas entre os grupos pelo teste de Qui-quadrado. Foi considerado significativo um $p<0,05$.

\section{RESULTADOS}

No período de janeiro a dezembro de 2016, 355 neonatos foram internarados na UTIN, onde 69 foram selecionados para o estudo. Destes, 29 foram excluídos por não se encaixarem nos critérios de inclusão. Por fim, a amostra foi constituída por 40 registros de nascimentos, divididos em parto vaginal
27,5\% ( $n=11)$ e cesárea 72,5\% ( $n=29)$ (Figura 1 ), com SDR, o que representa $11 \%$ dos pacientes internados no referido período.

Outras patologias: malformação congênita (2), conjuntivite (1), hipoglicemia (1), icterícia (2), hipertensão pulmonar persistente (2), Diabetes Mellitus Gestacional (1), convulsões (1).

\section{Dados maternos}

A média geral de idade materna foi de 27 anos, com uma média de 9 consultas pré-natais. Dos 40 registros coletados, $40 \%(n=16)$ das mães tiveram alguma complicação durante a gestação e $45 \%(n=18)$ entraram em trabalho de parto. Na Tabela 1 os dados maternos estão apresentados de acordo com o tipo de parto.

Figura 1 - Fluxograma do estudo

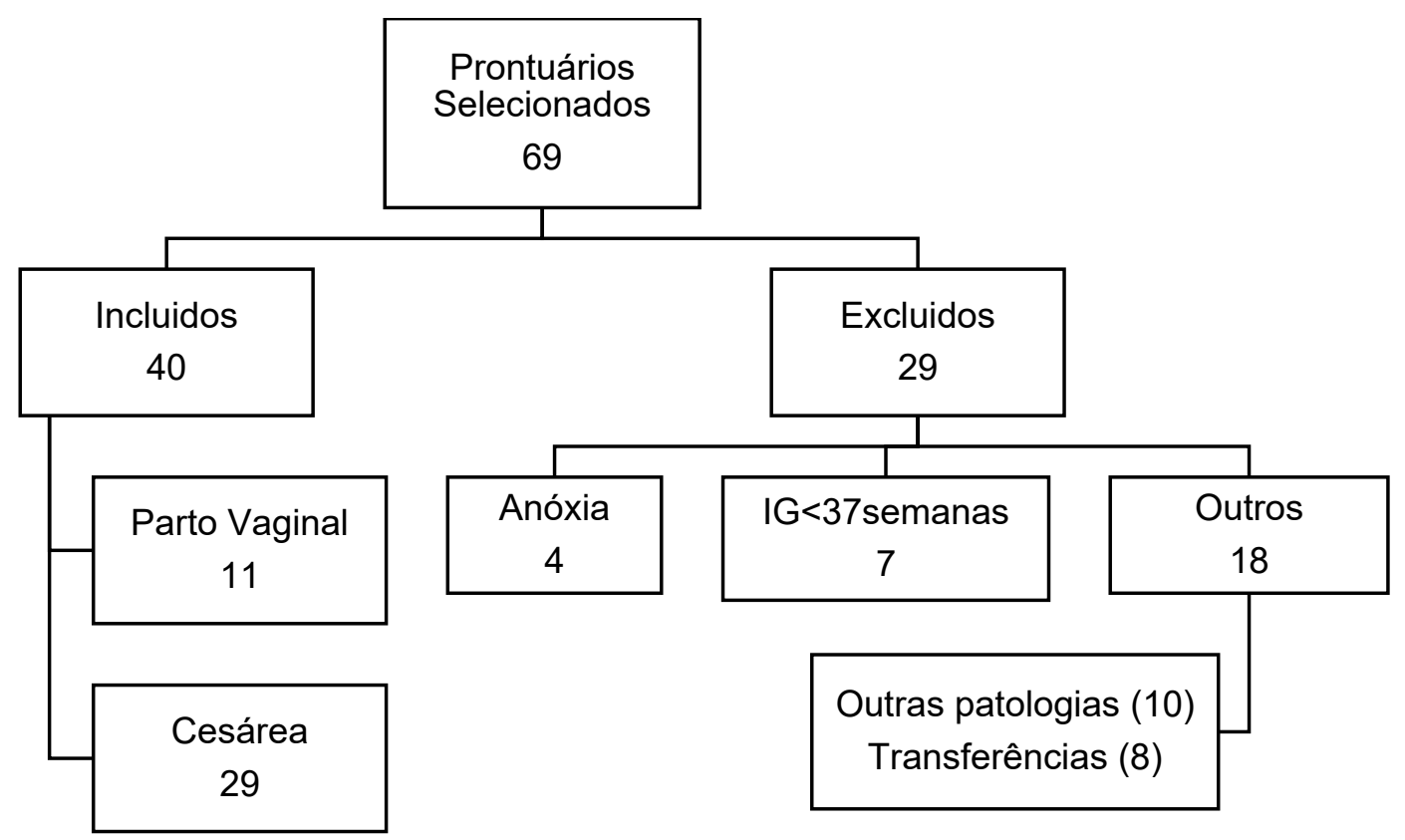

Outras patologias: malformação congênita (2), conjuntivite (1), hipoglicemia (1), icterícia (2), hipertensão pulmonar persistente (2), Diabetes Mellitus Gestacional (1), convulsões (1). 
Tabela 1 - Características maternas

\begin{tabular}{lccc}
\hline & $\begin{array}{c}\text { Parto Vaginal } \\
(\mathrm{n}=11)\end{array}$ & $\begin{array}{c}\text { Cesárea } \\
(\mathrm{n}=29)\end{array}$ & p-valor \\
\hline Idade (anos) & $27,0(24,0-34,0)$ & $25,0(19,5-29,0)$ & 0,369 \\
\hline Gestações anteriores & $2,0(1,0-3,0)$ & $2,0(1,0-3,0)$ & 0,788 \\
\hline Partos anteriores & $1,0(0,0-3,0)$ & $1,0(1,0-2,5)$ & 0,788 \\
\hline Pré-natal & $5,0(0,0-9,0)$ & $7,0(3,0-11,5)$ & 0,280 \\
\hline $\begin{array}{l}\text { Complicações na } \\
\text { gestação }\end{array}$ & $0(0)$ & $3(10,3)$ & 0,267 \\
\hline Sífilis n (\%) & $0(0)$ & $1(3,4)$ & 0,533 \\
\hline Obesidade n (\%) & $1(9,1)$ & $5(17,2)$ & 0,519 \\
\hline DMG n (\%) & $0(0)$ & $1(3,4)$ & 0,533 \\
\hline Depressãon (\%) & $3(27,3)$ & $0(0)$ & $0,003^{*}$ \\
\hline ITUn (\%) & $0(0)$ & $1(3,4)$ & 0,533 \\
\hline Toxoplasmose n (\%) & $0(0)$ & $1(3,4)$ & 0,533 \\
\hline TVP n (\%) & $10(90,9)$ & $8(27,6)$ & $<0,001^{*}$ \\
\hline Trabalho de parto n (\%) & & & \\
\hline
\end{tabular}

DMG: Diabetes Mellitus Gestacional; ITU: Infecção do Trato Urinário; TVP: Trombose Venosa Profunda.

Dados apresentados mediana e intervalo interquartil, frequência e porcentagem. $p<0,05$.

*: diferenças significativas entre os grupos.

\section{Dados neonatais no nascimento e durante a internação}

Dos 40 registros coletados, $62,5 \%(n=25)$ dos neonatos eram do sexo masculino, em média com 3.203 gramas e idade gestacional de 38 semanas. A média do índice de Apgar no primeiro minuto foi $7 \mathrm{e}$ no quinto minuto 9 . Dos 40 neonatos, $37,5 \%$ $(n=15)$ necessitaram de reanimação em sala de parto. Os dados neonatais do nascimento, de acordo com o tipo de parto, estão apresentados na Tabela 2.

Durante o período de internação $65 \%(n=26)$ dos 40 neonatos necessitaram de oxigenoterapia, 15\% $(n=6)$ de ventilação mecânica (VM) e 30\% ( $n=12)$ Pressão Positiva Contínua em Vias Aéreas (CPAP). O antibiótico foi necessário em 42,5\% $(n=17)$ dos casos. Os dados neonatais durante a internação estão expostos na Tabela 3 separados por tipo de parto. 
Tabela 2 - Características neonatais no nascimento

\begin{tabular}{|c|c|c|c|}
\hline & $\begin{array}{l}\text { Parto Vaginal } \\
\quad(n=11)\end{array}$ & $\begin{array}{c}\text { Cesárea } \\
(n=29)\end{array}$ & p-valor \\
\hline Sexo masculino $\mathrm{n}(\%)$ & $7(63,6)$ & $18(62,1)$ & 0,927 \\
\hline Peso (Kg) & $3,2(2,7-3,8)$ & $3,1(2,8-3,3)$ & 0,419 \\
\hline IG (semanas) & $38,0(37,0-39,0)$ & $38,0(37,0-39,0)$ & 0,698 \\
\hline Apgar $1^{\circ} \mathrm{min}$ & $8,0(7,0-9,0)$ & $8,0(6,0-8,0)$ & 0,437 \\
\hline Apgar $5^{\circ} \mathrm{min}$ & $9,(9,0-10,0)$ & $9,0(8,0-9,0)$ & 0,207 \\
\hline $\begin{array}{l}\text { Reanimação em sala de } \\
\text { parton }(\%)\end{array}$ & $1(9,1)$ & $14(48,3)$ & $0,046^{*}$ \\
\hline
\end{tabular}

IG: Idade Gestacional

Dados apresentados em média, desvio padrão, mediana e intervalo interquartil. $p<0,05$.

*: diferenças significativas entre os grupos.

Tabela 3 - Características neonatais durante a internação

\begin{tabular}{lccc}
\hline & $\begin{array}{c}\text { Parto Vaginal } \\
(\mathrm{n}=11)\end{array}$ & $\begin{array}{c}\text { Cesárea } \\
(\mathrm{n}=29)\end{array}$ & p-valor \\
\hline Oxigenoterapia $\mathrm{n}(\%)$ & $9(81,8)$ & $17(58,6)$ & 0,170 \\
\hline Uso de VM n (\%) & $2(18,2)$ & $4(13,8)$ & 0,729 \\
\hline Uso de CPAP n (\%) & $3(27,3)$ & $9(31,0)$ & 0,817 \\
\hline Antibioticoterapia n (\%) & $6(54,5)$ & $11(37,9)$ & 0,343 \\
Fisioterapia n (\%) & $11(100)$ & $29(100)$ & 1,000 \\
\hline Tempo de internação (dias) & $7,0(4,0-11,0)$ & $6,0(2,0-11,0)$ & 0,437 \\
\hline
\end{tabular}

VM: Ventilação Mecânica; CPAP: Pressão Positiva Contínua em Vias Aéreas

Dados apresentados em frequência, porcentagem, mediana e intervalo interquartil. $p<0,05$. 


\section{DISCUSSÃO}

Este estudo analisou retrospectivamente as características de neonatos com SDR admitidos em uma UTIN, levando em consideração o tipo de parto, bem como fatores maternos que podem contribuir com a SDR, número consultas pré-natais e o curso durante a internação.

Dentre os fatores de risco para SDR está a cesariana ${ }^{8}$, especialmente pela ausência de compressão torácica, mecanismo que auxilia na excursão do liquido pulmonar e, quando realizada de forma eletiva, a baixa concentração de surfactante ${ }^{9}$. A cesariana, ainda, expõe o recém-nascido a maiores riscos de admissão em UTIN ${ }^{10}$ and even save lives. But the past two decades have brought a sharp growth in caesareans in many nations, raising concerns about unnecessarily high rates. Caesarean delivery on maternal request is relatively rare in the UK $(1-2 \%$ of births, o que foi sugerido em nosso estudo, onde grande parte dos neonatos nasceram de cesariana $(72,5 \%)$.

Alguns fatores maternos podem corroborar para que o neonato desenvolva a SDR, dentre eles estão a primigestação, complicações na gestação e doenças crônicas ${ }^{8}$. Assim como Cardoso, Alberti, Petroianu ${ }^{11}$ no presente estudo, em ambos os grupos, não houve maior prevalência de mães primigestas. Quanto as complicações gestacionais, de um modo geral, não foram observadas diferença, apenas para infecção do trato urinário (ITU), onde todos neonatos que nasceram de parto vaginal se internaram na UTIN pela síndrome.

Segundo o Ministério da Saúde é preconizado a realização de, no mínimo, 6 consultas de acompanhamento pré-natal ${ }^{12}$. Em nosso estudo observamos uma média de 7 consultas pré-natais, ou seja, pouco mais do que o preconizado, contudo, $20 \%(n=8)$ das 40 parturientes não realizaram nenhuma consulta.

Em sua pesquisa, Damian et al. ${ }^{13} \mathrm{co}-$ mentam que estudos realizados na Região Sul do Brasil demonstram que as internações na UTIN tem como causa uma relação de fatores vinculados a atenção básica, especialmente, durante a gestação e o nascimento, afirmando que existe uma necessidade urgente de se repensar as consultas de pré-natal e o atendimento ao parto, demonstrando a identificação precoce de sinais de riscos ou alterações na gestação que possam levar a um parto prematuro e/ou com complicações neonatais.

Alguns fatores auxiliam na redução de riscos para desenvolver a SDR, como o trabalho de parto que acelera a reabsorção do líquido interalveolar, por meio de liberação de fatores humorais. Quando esse processo não é desencadeado, a liberação de catecolaminas e corticosteroides (obrigatórios para maturação final dos pulmões do neonato) também não ocorre, aumentando a chance de patologias pulmonares ${ }^{4,14} 2013$, at $37-38+6$ weeks of pregnancy. The women were divided into exposed group (Group A. O que pode ser sugerido pelo presente estudo, onde apenas $7,6 \%$ dos neonatos que nasceram de cesariana passaram pelo trabalho de parto.

Corroborando, em maio de 2016, o Conselho Federal de Medicina ${ }^{15}$ preconizou que, em situações de risco habitual, a cesariana eletiva, isto é, sem o trabalho de parto, deve ser realizada a partir da $39^{a}$ semana de 
gestação ${ }^{4,15} 2013$, at 37-38+6 weeks of pregnancy. The women were divided into exposed group (Group A, com o intuito de garantir a segurança e saúde do neonato ${ }^{15}$. Em nosso estudo, ao contrário do que foi preconizado, $55 \%(n=16)$ dos neonatos que nasceram de cesariana tinham IG abaixo de 39 semanas e não realizaram trabalho de parto.

Quando falamos em fatores de riscos relacionados ao neonato os mais citados são o sexo masculino, baixos índice de Apgar e baixo peso ao nascimento ${ }^{3,4,16}$ Korea. Corroborando, com o nosso estudo teve, em sua amostra, $62,5 \%$ neonatos do sexo masculino (7 de parto vaginal e 18 de cesariana) fator que é vinculado a um processo de maturidade pulmonar mais lento durante o crescimento fetal resultando em uma maior fragilidade, devendo ser observado durante seu desenvolvimento também na vida extrauterina ${ }^{17}$. Fatores como índice de Apgar e peso apresentaram-se normais no presente estudo, sem diferenças significativas em ambos os grupos.

Uma pequena parte desses neonatos requer ajuda para fazer a transição intrauterina para extrauterina. Quando necessitam de manobras, raramente se trata de reanimação cardiopulmonar ou uso de drogas para reestabelecer a vitalidade fetal, pois a grande maioria necessita de ventilação por pressão positiva (VPP) ${ }^{15}$. No presente estudo, quando relacionado necessidade de reanimação em sala de parto com o tipo de parto, houve uma diferença significativa ( $p=0,046)$, ou seja, neonatos que nasceram de cesariana necessitaram mais $(48,3 \%)$ de reanimação em sala de parto quando em comparação ao parto vaginal $(9,1 \%)$. Resultados que vão ao encontro do estudo de Cancelier et al. ${ }^{18}$, que encontraram neonatos de parto cesáreo $(61,6 \%)$ com risco duas vezes maior de necessidade de reanimação do que nascidos de parto normal.

Juntamente com tais condições/curso do nascimento dos neonatos, a SDR, que apesar de ser uma doença benigna, muitas vezes vem associada à hipoxemia e à insuficiência respiratória, fatores que aumentam a necessidade de suporte ventilatório ${ }^{19} \mathrm{respec}-$ tively. Demographic, clinical and laboratory characteristics were compared between the two groups. Patients in group 2 had lower gestational age, higher Silverman and $\mathrm{Ri}-$ chardson scores, longer mean duration of oxygen support and hospitalization. A positive correlation was found between subcostal and xiphoid retractions, asynchrony in chest-abdomen movements, arterial $\mathrm{pH}<7.30$, ratio of $\mathrm{PaO}<$ sub $>2</$ sub $>1 \%$ inspired $\mathrm{O}<$ sub $>2</$ sub $><1.2$ and need of respiratory support $(\mathrm{p}<0.05$. Conforme Kahvecioğlu et al. ${ }^{19}$ respectively. Demographic, clinical and laboratory characteristics were compared between the two groups. Patients in group 2 had lower gestational age, higher Silverman and Richardson scores, longer mean duration of oxygen support and hospitalization. A positive correlation was found between subcostal and xiphoid retractions, asynchrony in chest-abdomen movements, arterial $\mathrm{pH}<7.30$, ratio of $\mathrm{PaO}<$ sub $>2</$ sub $>1 \%$ inspired $\mathrm{O}<$ sub $>2</$ sub $><1.2$ and need of respiratory support $(p<0.05$ desenvolveram um estudo com objetivo de identificar fatores que possam auxiliar a prever a gravidade da taquipneia transitória do recém-nascido e, assim, na possível necessidade de suporte respiratório e tiveram como achados que metade $(49,4 \%)$ da amostra necessitaram de oxigenoterapia. 
De encontro, com o nosso estudo, mais da metade dos neonatos (65\%) fizeram uso de oxigenoterapia, por $\mathrm{CENO}_{2}$ (Cateter endonasal), durante a permanência na UTIN, sendo a maior necessidade naqueles que nasceram de parto vaginal $(81,8 \%)$ do que cesariana $(58,6 \%)$.

Entretanto, a necessidade de um suporte ventilatório mais eficaz pode ser necessário, visto que esses neonatos podem ter sinais que duram de algumas horas até vários dias, podendo esse ser realizado através do CPAP ou $\mathrm{VM}^{20}$. No presente estudo, apenas $45 \%$ necessitou de uma destas modalidades, sendo $15 \%$ VM e 30\% CPAP nasal sem diferenças entre os grupos. O que podemos complementar com os achados quanto ao tempo de internação, que foi, em média, 8,48 dias para o total da amostra. Sugerindo que quanto maior o tempo de permanência na UTIN maior é a necessidade do suporte ventilatório mais eficaz, entretanto, não foi possível realizar uma análise de correlação.

Contudo, o ambiente hospitalar pode oferecer riscos de infecção para esses neonatos decorrente da imaturidade do sistema respiratório e a grande vulnerabilidade, sendo esta uma causa importante de óbitos em países desenvolvidos. As infecções na UTIN pode ser de forma precoce (até 48 horas) ou tardia (após 48 horas), diante disso a ampicilina e gentamicina são os antibióticos indicados para tratamento em infecções precoces ${ }^{13}$. Em seu estudo Granzotto et al. ${ }^{21}$ encontrou, ao investigar fatores de mortalidade e condições perinatais de neonatos internados em uma UTIN de Pelotas/RS, o uso de antibiótico em $80,2 \%$ dos pacientes. O presente estudo teve apenas 17 (42,5\%) neonatos com uso de antibiótico, fato este, que se deve a preocupação da equipe da unidade estudada, em um uso de forma racional e segura de antibiótico, sendo a SDR, de forma isolada, uma condição que não necessita desta intervenção.

Quanto a prevalência da SDR nas UTIN, uma pesquisa realizada em uma UTIN de um hospital de Recife-PE encontrou prevalência de $98,8 \%$ de recém- nascidos com $\mathrm{SDR}^{22}$. Em nosso estudo $11 \%$ das internações no ano de 2016 foram pela SDR. Diante da epidemiologia da doença destacamos a necessidade do fisioterapeuta inserido na UTIN em período integral, por contribuírem para reduzir a morbidade neonatal, o tempo de hospitalização, os custos hospitalares e as complicações $^{1,23}$, bem como nas unidades de atenção básica, com estratégias multiprofissionais de prevenção e de redução de fatores de riscos para o neonato.

Como limitação do estudo identificou-se a falta de dados sobre neonatos com as mesmas características, porém, que não foram internados na UTIN, ou seja, que não apresentaram a SDR (grupo controle). Assim, seria possível traçar o perfil dos mesmos, apontando seus principais fatores de risco, tanto maternos, gestacionais quanto de via de nascimento. Outro fator a ser destacado é que diante de uma amostra maior tais fatores seriam melhor evidenciados. Futuros estudos podem ser realizados de forma multicêntrica, como consequência uma amostra maior.

\section{CONCLUSÃO}

Sendo assim, a prevalência dos neo- 
natos com SDR admitidos eram do sexo masculino, com apgar normal e que necessitaram de suporte ventilatório durante a internação. Quanto ao tipo de parto, tivemos um predomínio de neonatos nascidos de cesariana, sem a presença do trabalho de parto e com idade gestacional de 38 semanas. Ressaltamos que, caso a preconização do Conselho Federal de Medicina fosse devidamente cumprida,

\section{REFERÊNCIAS}

1. Brasil. Ministério da Saúde. Agência Nacional de Vigilância Sanitária. Resolução-RDC no 7 , de 24 de fevereiro de 2010.

2. Liszewski MC, Stanescu AL, Phillips GS, Lee EY. Respiratory Distress in Neonates: Underlying Causes and Current Imaging Assessment. Radiol Clin North Am 2017; 55(4):629-644.

3. Kim BB, Chung S-H, Yoon H-S, Hahn W-H, Bae C-W, Choi Y-S. Decreased Cystatin C-Estimated Glomerular Filtration Rate Is Correlated with Prolonged Hospital Stay in Transient Tachypnea of Newborn Infants. Pediatr Neonatol 2016; 57(3):195-200.

4. Dileep A, Khan NB, Sheikh SS. Comparing neonatal respiratory morbidity in neonates delivered at term by elective Caesarean section with and without dexamethasone: Retrospective cohort study. J Pak Med Assoc 2015; 65(6):607-611.

5. Furzán JA. Nacimiento por cesárea y pronóstico neonatal. Arch Venez Pueric Pediatr 2014; 77(2):79-86.

6. Santana SMP, Novais MAP, Zucchi P. Internações Hospitalares de Neonatos com Síndrome do Desconforto Respiratório e sua Participação nas Internações Hospitalares Neonatais no Âmbito do Sistema Único de Saúde em 2015. Int J Heal Manag Rev 2016; 2(1):1-18.

7. Pramanik AK, Rangaswamy N, Gates T. Neonatal Respiratory Distress: A Practical Approach to Its Diagnosis and Management. Pediatr Clin North Am 2015; 62(2):453-469.

8. Pérez-Cuevas R, Jasso Gutiérrez L, Doubova S, Flores Hernández S, Mantilla Trollé C, González Guerra E, et al. Evaluación de la calidad de la atención de la taquipnea transitoria en recién nacidos afiliados al Seguro Médico Siglo XXI. Bol Med Hosp Infant Mex 2014; 71(6):346-351.

9. Oliva CMPS, Dias C. Cesariana versus Parto Vaginal : como nascer?, https://repositorio-aberto.up.pt/bitstream/10216/52793/2/Tese de mestrado.pdf (2010).

10. Blustein J, Liu J. Time to consider the risks of caesarean delivery for long term child health. Bmj 2015; 350(jun09 3):h2410-h2410.

11. Cardoso PO, Alberti LR, Petroianu A. Morbidade neonatal e maternas relacionada ao tipo de parto. Cien Saude Colet 2010; 15(2):427-435.

12. Ministério da Saúde. PORTARIA № 570, DE $1^{\circ}$ DE JUNHO DE 2000, http://bvsms.saude.gov.br/bvs/saudelegis/ gm/2000/prt0570_01_06_2000_rep.html (2000, accessed 2 April 2019). mais da metade das internações de neonatos nascidos de cesariana seriam potencialmente evitáveis.

Por fim, destacamos a necessidade de estratégias multidisciplinares desde a atenção básica, bem como estudos que possam subsidiar as reflexões e condutas diante dos fatores de risco tanto maternos, de parto e, posteriormente, neonatais.

13. Damian A, Waterkemper R, Paludo CA. Perfil de neonatos internados em unidade de tratamento intensivo neonatal : estudo transversal. Arq Ciências da Saúde 2016; 23(2):100-105.

14. Sadeck $L$ dos $S R$. Diagnóstico diferencial das insuficiências respiratórias agudas. Rev Med 2003; 82(1-4):40-45.

15. Lima CVTC, Silva HBE. Diário Oficial da União - Conselho Federal de Medicina. Applied Soil Ecology 2016; 138.

16. Celebi MY, Alan S, Kahvecioglu D, Cakir U, Yildiz D, Erdeve O, et al. Impact of Prophylactic Continuous Positive Airway Pressure on Transient Tachypnea of the Newborn and Neonatal Intensive Care Admission in Newborns Delivered by Elective Cesarean Section. Am J Perinatol 2016; 33(1):99-106.

17. Souza KCL de, Campos NG, Júnior FFUS. Perfil dos recém-nascidos submetidos à estimulação precoce em uma unidade de terapia intensiva neonatal. Rev Bras em Promoção da Saúde 2013; 26(4):523-529.

18. Cancelier ACL, Anesi S, Dequi PW, Silva MF da. Resuscitation maneuvers in the delivery room: analysis of care of newborns. Arq Catarin Med 2014; 43(4):44-49.

19. Kahvecioğlu D, Çakır U, Yıldız D, Alan S, Erdeve Ö, Atasay B, et al. Transient tachypnea of the newborn: Are there bedside clues for predicting the need of ventilation support? Turk J Pediatr 2016; 58(4):400-405.

20. Aathi MK. Transient Tachypnea of Newborn ( TTN ): An Overview. Int J Nurs Educ Res 2014; 2(2):99-103.

21. Granzotto JA, Stringari Da Fonseca S, Lindemann FL. Fatores relacionados com a mortalidade neonatal em uma Unidade de Terapia Intensiva neonatal na região Sul do Brasil. Rev da AMRIGS 2012; 56(1):57-62.

22. Vasconcelos GAR De, Almeida RDCA, Bezerra ADL. Repercussões da fisioterapia na unidade de terapia intensiva neonatal. Fisioter em Mov 2011; 24(1):65-73.

23. Nicolau, Carla Marques; Lahóz AL. Fisioterapia respiratória em terapia intensiva pediátrica e neonatal: uma revisão baseada em evidências. Pediatr (Säo Paulo) 2007; 29(3):216-221.

\section{CORRESPONDÊNCIA}

Adriane Schmidt Pasqualoto

Av. Roraima, 1000, Prédio 26D, sala 1441, Cidade Universitária, Bairro Camobi, Santa Maria - RS, CEP: 97105-900,

Universidade Federal de Santa Maria. 